\title{
Desafíos Epistemológicos de la Identidad Cultural en el Contexto Universitario
}

\section{Epistemological Challenges of Cultural Identity in the University Context}

* Segundo Ángel Caiza Tisalema ${ }^{1}$,**Melquiades Mendoza Pérez², ***Raúl Bolívar Cárdenas Quintana ${ }^{2}, * * * *$ Luís Gózalo López Rodríguez ${ }^{2}$,****Segundo Adolfo Bassante Jiménez

${ }^{1}$ Lic. Maestrante en Educación, Innovación en Liderazgo Educativo, Universidad Tecnológica Indoamérica, ${ }^{2}$ Docente Investigador Universidad Técnica de Cotopaxi

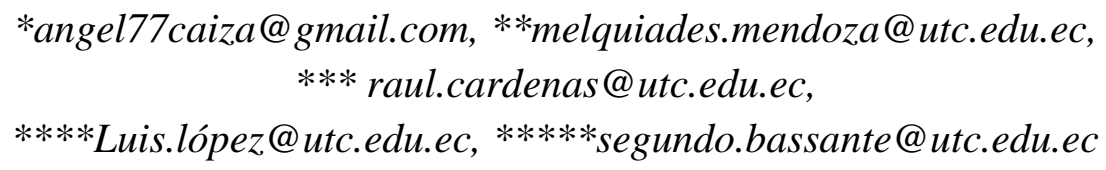

Fecha de recepción: 11/07/2018

Fecha de aceptación: 21/08/2018

Publicado: 26/12/2018

\section{Resumen}

Asumir que el contexto universitario representa un desafío teórico es anclarse en una postura científica explicativa para comprender la identidad y derivar en consecuencia comportamientos donde se logre fundamentar categorías y nociones que enriquezcan un campo de ciencia. Reto que aspira a desarrollar el presente artículo; mediado por el método hermenéutico textual, se establece como aporte un nivel reflexivo que brinda guías para conocer el sentido y el valor de la actividad intelectual. Al aportar un nivel reflexivo que brinde guías para conocer el sentido y el valor de la actividad intelectual desde la identidad en la universidad, se enriquece el conocimiento de la concepción y a la vez de los escenarios de la academia. Este estudio ha demostrado entonces que el diálogo de lo epistemológico en las relaciones de la coherencia conceptual tiene su efecto en el contexto que lo especifica. Una aseveración que trascurre por las intersecciones que estructuran la ciencia cultural, para enriquecer al sujeto y disponerlo en condiciones de expresar sus sentimientos y racionalidades desde el compromiso con su realidad más inmediata. En el entorno de la identidad se impacta la diversidad cultural, un factor que por la labor de instituciones y organismos ha sido posible 
recuperar aquello que se daba por perdido para poder apreciarlo y valorarlo. La Universidad es la sede de la ciencia y de la razón cuyas relaciones interétnicas e interculturales de mutuo respeto y dignidad proporcionan una identidad positiva a los sujetos sociales.

Palabras clave: desafíos epistemológicos, identidad, contexto universitario

\begin{abstract}
Assuming that the university context represents a theoretical challenge is to anchor oneself in an explanatory scientific position to understand the identity and consequently derive behaviors where it is possible to base categories and notions that enrich a field of science. Challenge that aspires to develop the present article; mediated by the textual hermeneutic method, it is established as a contribution a reflective level that provides guidelines to know the meaning and value of intellectual activity. By providing a reflective level that provides guides to know the meaning and value of intellectual activity from the identity in the university, the knowledge of the conception and at the same time of the scenarios of the academy is enriched. This study has shown then that the dialogue of the epistemological in the relations of conceptual coherence has its effect in the context that specifies it. An assertion that goes through the intersections that structure cultural science, to enrich the subject and arrange it in conditions to express their feelings and rationalities from the commitment to their immediate reality. In the environment of identity, cultural diversity is impacted, a factor that through the work of institutions and organizations it has been possible to recover what was considered lost in order to appreciate and value it. The University is the seat of science and reason whose interethnic and intercultural relations of mutual respect and dignity provide a positive identity to social subjects.
\end{abstract}

Keywords: epistemological challenges, identity, university context 


\section{Introducción}

Asumir que el contexto universitario representa un desafío teórico es anclarse en una postura científica explicativa para comprender la identidad y derivar en consecuencia comportamientos donde se logre fundamentar categorías y nociones que enriquezcan un campo de ciencia. Reto que aspira a desarrollar el presente artículo; mediado por el método hermenéutico textual, establece como aporte un nivel reflexivo que brinda guías para conocer el sentido y el valor de la actividad intelectual.

Su posicionamiento se inscribe en la visión de que la epistemología tiene por objeto ese conocimiento que se soporta en sí mismo o que soporta alguna disciplina en su especificidad; lo que la sustenta como tal, su esencia, sus alcances y límites en su acepción interna, propia de la disciplina, y externa, su influencia en el contexto social (Jaramillo, 2003). Desde este soporte es argumentado el devenir del trabajo que aspira a establecer que los retos de la identidad en el orden epistemológico son un continum entre la acepción interna y su determinación contextual.

La identidad en tanto proceso, está socialmente estructurada en razón de que en sus niveles los individuos delimitan fronteras con otros grupos o sujetos con la intencionalidad explícita de producir una diferenciación respecto a grupos con los cuales interaccionan (Giménez, 2002: 60). Por ello, la identidad es una construcción social dentro de una situación relacional, es decir, una configuración que emerge de la interacción y comunicación social, lo cual orienta sus representaciones y acciones (Giménez, 2002: 60). Esta aspiración de definir la identidad en tanto construcciones simbólicas resulta un involucramiento de representaciones y clasificaciones referidas a las relaciones sociales y las prácticas, donde se juega la pertenencia y la posición relativa de personas y de grupos en su mundo (Bayardo, 2001). Con lo cual se demuestra que en la vida cotidiana esta adquiere sentido. 


\section{Desarrollo}

Esta postura constructivista ha predominado en su precisión terminológica, lo cual no excluye otras formas de visualización que emergen de diferentes ámbitos epistemológicos. Así pueden localizarse posturas que hablan de conflictos identitarios debido a contradicciones entre colectividades e individuos donde se juega la pertenencia y la posición relativa de personas y de grupos en su mundo (Bayardo, 2001). Esa circunstancia arriesgada es un resultado de incorporaciones y obviedades. Los nuevos enfoques acerca de la identidad, en estrecha vinculación con los planteos previos, enfatizan su carácter plural, cambiante, constituido en los procesos de lucha por el reconocimiento social.

La delimitación frente a otro conjunto de actores requiere de una comparación y selección -realizada de forma consciente- de determinadas prácticas y representaciones como “emblemas de contraste" (Giménez, 1994:172). Estos "emblemas de contraste" son reconocidos por los actores, tanto por los del propio grupo como por los del resto. Es lo que este autor menciona como "“auto-identidad' y 'exo-identidad”, es decir, de autoafirmación y de asignación, que tienen lugar dentro de dinámicas socio-políticas determinadas en la lucha por la "clasificación legítima" (Giménez, 2002: 61). Una conceptualización que incluye una toma de decisiones metodológicas, que eviten visualizarla como un concepto fijo puesto que se recrea individual y colectivamente; además se alimenta de la influencia del medio exterior. Con los estudios antropológicos y sociológicos la identidad asume una visión donde se señala la reafirmación frente al otro.

Es innegable la existencia de una identidad personal o individual y colectiva, por la cual se definirá cada persona como autónomo, étnico, comunitario, donde se adquiere tal carácter. Esta colectividad puede estar localizada de manera geográfica, pero no necesariamente, como sucede en el caso de los refugiados. La colectividad, entonces, también 
se evidencia de otras formas, como es el caso de las manifestaciones culturales. Estévez (2002) señala al respecto que la identidad es la respuesta a la pregunta quién soy; a nivel individual, o quienes somos; a nivel grupal, étnico, nacional o continental. Las escalas descritas indican las diversas maneras en que es posible localizarla. El análisis realizado de diferentes autores ante mencionados, conceptualiza que la identidad, se define por características y cualidades que llevan los seres humanos para ser diferentes de uno al otro, por cual es necesario identificar quienes somos y a dónde vamos.

\section{Identidad cultural.}

La identidad también puede manifestarse a través de la cultura, que puede ser conceptualizada como un nivel específico y necesario de toda práctica humana propuesto por Néstor García Canclini (1991), hecho que señala la dimensión simbólica que la constituye y donde están presentes lo económico junto a lo social y la posibilidad analítica de distinguirla y no apreciarla como simple reflejo o expresión.

En este trabajo, esa postura surge de la comprensión de los problemas resueltos por el campo disciplinar acerca de la transversalización de la ciencia, entre los cuales se sitúa la relación entre la semiótica, la comunicación y la sociología de la cultura. En esta complejidad los Estudios Culturales saldan los errores al estudiar la relatividad de estas concepciones como intersecciones donde deben analizarse los efectos co-determinantes entre uno y otro plano del análisis. Además es preciso agregar que los ámbitos de la identidad cultural no son representaciones estáticas, si no entidades sujetas a cambios permanentes, condicionados por factores externos.

La temática de la identidad cultural implica la conjugación por tanto de la mismidad y la simbolización que da lugar a esa identificación común. Montaluisa \& Sanchez, (2016) en relación con esa visión apelan al Diccionario de la Lengua Española, para localizar la Cultura 
como: "Resultado o efecto de cultivar los conocimientos humanos y de afinarse por medio del ejercicio, las facultades intelectuales del hombre". De acuerdo con las definiciones de los autores tiene una correlación en sus conceptos; la cultura comprende los conocimientos vivenciales de un grupo humano, situados en las comunidades, pueblos, ciudades y continentes.

Sin olvidar que la cultura es casi una abstracción obtenida de la observación del comportamiento y de las relaciones de los individuos entre ellos, es conveniente indicar algunas características de la cultura:

1. La cultura se aprende y se trasmite. Mediante los procesos de socialización a los individuos, a través de diversos culturales propios. La lengua y los sistemas de valores, los gustos de costumbres, las maneras de entender el mundo y la vida, las formas de organización política económica y otros.

2. La cultura es esencialmente simbólica. La lengua es uno de los aspectos con más carga simbólica y también uno de los elementos que hace posible la continuidad de las culturas. Que permite conocer su forma de comunicación con otras culturas.

3. La cultura es un sistema. Los elementos que configuran una cultura forma parte de un todo, y desde esta perspectiva de totalidad tiene sentido.

La cultura finalmente es, dinámica porque su funcionalidad requiere de la capacidad de cambio para poder responder a las transformaciones contemporáneas que se suscitan en el mundo. En este contexto impactan los movimientos migratorios, en tanto propiciadores de reconfiguraciones culturales en diferentes ámbitos sociales. Incluye el conjunto de prácticas, actitudes, valores, idioma, tradiciones, costumbres, comportamientos propios de un grupo colectivo, factores que se conjugan y comprenden como determinantes de la identidad cultural. 
De este modo, si bien es cierto existe diversas definiciones, en general predomina la percepción que cultura es lo que da vida al ser humano. Es posible entonces decir que la cultura tiene varias dimensiones y funciones sociales.

Resulta necesario acotar que cada persona estamos dentro de una cultura particular, aprendida de los padres y ellos de sus ancestros. Contextualizando esta visión en Ecuador permite integrar junto a su gran riqueza económica y de recursos materiales, la cultural cuya mejor expresión se sintetiza en la cosmovisión andina de la Pacha mama "Madre tierra".

A propósito de ello, Montaluisa \& Sánchez,2016) define la identidad cultural de la siguientes manera: aspectos en los que se plasma su cultura, como lengua, instrumento de comunicación entre los miembros de la comunidad, las relaciones sociales, ritos, ceremonias propias, o comportamientos colectivos, esto es sistema de valores y creencias. De la misma manera señala que la identidad cultural significa: conjunto de valores, tradiciones, símbolos, creencias, modos de comportamiento, que funciona como elemento cohesionador dentro de un grupo social.

Esta dinámica abarcadora puede ser precisada, así Sáez, (2013) menciona que la identidad cultural: son procesos socio psicológicos que parte de la identificación de un grupo humano con aquellos elementos tangibles que son: poesías, ritos, medicina tradicional, música, danza, gastronomía, leyendas y otros. Hecho que implica el auto reconocimiento de un grupo humano de un pueblo, una nación y continente, a través de las condiciones históricas.

Funcionalidad histórica que Sánchez \& Dalama (2012) atribuyen a la importancia que la identidad cultural tiene al dar sentido y finalidad a la vida de una sociedad, que viene en generación (abuelos, padres, hijos,) estableciendo un vínculo entre pasado, el presente, y el futuro. Así también valorar formas de vida de los pueblos. 
En cambio otros autores como Odello (2012) obvian esa trascendencia y la aprecian como conjuntos de vidas de costumbres, conocimientos y grado de desarrollo artístico, en una época o grupo social. Giménez (2012) aporta que la identidad cultural determina las interrelaciones de la sociología y la antropología.

Esas lecturas conducen a concebir la identidad cultural desde su amplitud, y abarcamiento multidisciplinario ya sea la Antropología, la Sociología, la Historia, y su reflejo espacio y temporal.

Los presupuestos presentados anteriormente permiten al estudio de la identidad cultural evaluar el sentido de las diversas colectividades y su conciencia de cuáles son sus representaciones culturales, un tema fundamental en el país y urgido de reflexiones, a pesar de la complejidad y desafíos que supone realizarlo.

\section{Sobre las circunstancias de la identidad}

En los últimos años se ha visto en las comunidades indígenas fracturas en sus prácticas culturales con los consecuentes daños a la identidad cultural ecuatoriana, por diferentes circunstancias, y en relación a su indumentaria, idioma, costumbres, tradiciones, rituales.

Los problemas socio económicos, migración, educación, trabajo, por lo tanto han llevado a la aculturación de los pueblo como Tomabela. Estos factores inciden en la perdida de la identidad, muchos miembros de las comunidades indígenas han perdido los conocimientos ancestrales.

Día a día se viven y testimonian los procesos de aculturación en diferentes niveles y diferentes espacios, proceso donde tiene lugar un intercambio de elementos culturales entre distintos grupos sociales, que pueden estar generando la pérdida de la identidad cultural de los pueblos indígenas del Ecuador, es una realidad que se vive en los últimos 20 años; sin 
embargo, las raíces ancestrales se transforman en la base para la supervivencia en el modo de vida cotidiana.

Los pueblos indígenas, por trascendencia tienen un legado cultural que es más esforzado conservarlo, cual herencia que trasciende de generación en generación. Representa un reto sistematizar las prácticas religiosas, las costumbres que han servido para constituirse en una civilización, las actividades comunitarias como son las mingas, el maki mañachi, el randinchi, la kuyana, los ritos, el idioma y sobre todo la vestimenta características de cada pueblo.

En muchos pueblos indígenas del Ecuador ésta latente, con unos pueblos menos y con otros con mayor fuerza; QUE sin embargo, hay algunos que han conseguido sostener sus rasgos identitarios dentro de su comunidad, han logrado perseverar el campo artesanal, en la música, en lo intelectual, en el proceso organizativo, alcanzando el progreso.

Así mismo, en algunos pueblos es muy marcada la pérdida de la identidad y una confusión cultural al perder sus verdaderos símbolos que los identifican como propios de un pueblo. En la Unidad Educativa Intercultural Bilingüe "Huayna Capac" es posible constatar este proceso de quiebre de la identidad cultural en sus costumbres, tradiciones, idioma kichwa, vestimenta.

Según UNESCO la amplia difusión de la cultura y la educación de la humanidad para la justicia, la libertad y la paz son indispensables a la dignidad del hombre y constituyen un deber sagrado que todas las naciones han de cumplir con un espíritu de responsabilidad y de ayuda mutua.

Los Estados miembros asumen un compromiso de tomar las medidas apropiadas para difundir ampliamente la Declaración Universal de la UNESCO sobre la Diversidad Cultural y fomentar su aplicación efectiva, cooperando en particular con miras a la realización de los siguientes objetivos: 
"Salvaguardar el patrimonio lingüístico de la humanidad y apoyar la expresión, la creación y la difusión en el mayor el mayor número posible de lenguas."

"Fomentar la diversidad lingüística respetando la lengua materna, mediante la educación de los programas escolares y la formación de los docentes”. (UNESCO)

La conferencia general de la Organización Internacional del Trabajo (OIT) en el convenio sobre pueblos indígenas tribales 169, adoptó la Declaración Universal de los Derechos Humanos y el Pacto Internacional de los derechos económicos, sociales, culturales. Reconociendo así las aspiraciones de sus pueblos a asumir el control de sus propias formas de vida y fortalecer su identidad cultural.

La Constitución de la República del Ecuador en su artículo 21 dice: “Las personas tienen derecho a construir y mantener su propia identidad cultural, a decidir sobre su pertenencia a una o varias comunidades culturales y a expresar dichas elecciones; a la libertad estética; a conocer la memoria histórica de sus culturas y a acceder a su patrimonio cultural; a difundir sus propias expresiones culturales y tener acceso a expresiones culturales diversas." (Constitución de la República del Ecuador, 2008)

La Ley Orgánica de Educación Intercultural (LOEI) ecuatoriana, garantiza, valora y vela por el cumplimiento de la interculturalidad; a través una identidad propia de cada ser humano; y encarga este trabajo al Sistema Educativo y en especial a sus docentes; a través del proceso de enseñanza - aprendizaje, que busca el desarrollo holístico del ser humano y en especial la toma de conciencia moral y ética sobre sus diferencias individuales y culturales.

El Modelo del Sistema de Educación Intercultural Bilingüe (MOSEIB) permite fortalecer la calidad de la educación con pertinencia cultural y lingüística a fin de desarrollar las habilidades y destrezas cognitivas, psicomotrices y afectivas de los estudiantes de nacionalidades y pueblos en las instituciones educativas interculturales bilingües. (MOSEIB). 


\section{Diversidad cultural.}

Resulta importante subrayar la trascendencia de la riqueza cultural ecuatoriana. La diversidad cultural, si bien es cierto, que nace en tiempos remotos, pero tal y como lo describe Stainback (2001) desde la llegada de los españoles la interculturalidad se observa de manera un tanto dramática, cruel y despiadada, porque fueron ellos quienes han sometido a nuestros antepasados, sus valores, creencias, hábitos y conductas, opacando de una u otra forma los antecedentes culturales que se tenía; la cultura occidental intenta desplazar también nuestro origen, pero por la labor de instituciones y organismos ha sido posible recuperar aquello que se daba por perdido para poder apreciarlo y valorarlo, pues en los tiempos pretéritos no era penalizado un acto de burla de alguna persona de raza diferente (González de Alba, 2013).

En el siglo XX la inclusión de indígenas, blancos, mestizos y negros en el ámbito educacional propició la integración intercultural; años más tarde, después de la descolonización toma auge la cultura de los pueblos originarios; se impulsa así la interculturalidad porque de uno u otro modo es más fácil y común poder relacionarse con personas de cualquier etnia. (Valencia, 2012)

En el 2001 se reconoce la legislación promulgada por la UNESCO donde se busca rescatar y respetar la variedad de recursos naturales, ecológicos, y arqueológicos que ofrece cada país, el proyecto de Convención sobre la Diversidad Cultural elaborado por la Red Internacional de Políticas Culturales buscan ofrecer una respuesta concreta a los gobiernos, para poder seguir conservando la diversidad de los estados (UNESCO, 2004).

Diversidad cultural, según Jujuy (2015), es la variedad de diferentes culturas dentro de un grupo de personas o una sociedad, se toma en cuenta también la relación que se pueda dar entre dos o más culturas; esta diversidad cultural permite personas más cultas y tener una 
sociedad más respetuosa; con este propósito se declaró el día 21 de mayo el Día Mundial de la Diversidad Cultural para el Diálogo y el Desarrollo.

Tal como lo señala Arbat (2012), la diversidad cultural se involucra con la sociedad, la naturaleza y el medio ambiente, influenciando los sectores públicos o privados de las naciones, debido a que en cualquier lugar que una persona se encuentre va a encontrar una rica gama de culturas, razas, etnias, sujetos con costumbres diferentes a la suya.

La protección de la diversidad cultural desde el punto de vista político y económico se vuelve urgente en el contexto de la mundialización que amenaza con producir una desvalorización de las culturas originarias. En tal sentido se han producido cambios que pueden afectar o beneficiar a unos y a otros; aparentemente los países grandes se ven beneficiados por su reconocimiento y popularidad, los pequeños no tanto, aunque se puede considerar que cualquier país por pequeño y desconocido que sea puede contener una gran riqueza natural; tal y como es el caso de Ecuador (Ambrosi, Peugeot, Pimienta, 2005).

El Ecuador es un país pequeño en extensión territorial, pero grande en diversidad cultural y étnica, en beneficio de ello durante la presidencia de Rafael Correa, este crea un plan en donde se busca tener un mejor estilo de vida; en el 2008 entra en vigencia una ley que lo establece; se considera a Ecuador como un país intercultural y plurinacional, dada la gran cantidad de indígenas y afro ecuatorianos; aunque la mayor parte de la población es mestiza, todos son considerados hijos de este país sin distinción alguna, son un orgullo para la historia nacional (UNESCO, 2013). Esta diversidad humana se da en medio de una variedad de climas, espacios geográficos y realidades ambientales; lo cual no es solo una singularidad del país, sino también la base de la formación de identidades regionales (Universidad del Azuay, 2014).

Integración Social, Valores, Colaboración y Cooperación como factores determinantes de la preservación de la diversidad cultural ecuatoriana. 
Pérez y Merino (2011), afirman que la integración social es de carácter dinámico, activo y con muchos factores por la diferencia de los grupos que se encuentran en la sociedad y las múltiples diferencias étnicas, culturales, religiosas, entre otras. Pero sea cual sea todas tienen algún objetivo en común, en un mismo país pueden existir muchos tipos de integración social, comenzando por los niveles económicos, por ejemplo los de alta sociedad siempre se reúnen con su grupo, los de clase media igual y los pobres, o clase económica desfavorecida, de la misma manera. (Pere, 2009).

Arias, Arraiga, Gavia, Lillo y Yanes (2009), señalan que el objetivo de la integración social, es resaltar y alcanzar la igualdad entre las personas, dejando a un lado las barreras planteadas en sociedad; que se pueda vivir en conjunto con todos, dejando el racismo, los prejuicios sociales; así mismo fomentar el respeto y la tolerancia hacia las diferentes culturas. Al mencionar la integración, se toma en cuenta las culturas, las razas, los géneros, la edad, el sexo, las costumbres nativas o cualquier otro aspecto que se pueda identificar.

Según Romero (2013) unos de los principales exponentes o representantes de los estudios axiológicos fueron Platón y Aristóteles, pero a lo largo de la historia el pensamiento clásico axiológico ha sufrido transformaciones, y de muchas maneras se ha involucrado y relacionado con factores: económicos, sociales, políticos y religiosos. Junto con el surgimiento del Capitalismo en 1850 aparece el pensamiento filosófico de Max Scheler un enfoque praxiológico vinculado al ámbito burgués, donde se afirma con un enfoque reduccionista, que los valores son los que establecen la vida del ser humano. A este pensamiento se contrapone la opinión de León (2012) quien expone que los valores no existen si no hay una relación social, indica que los valores expresan las necesidades objetivas que tiene la sociedad.

Al tratar de definir los valores, Padrino (2009) afirma que son características buenas que el ser humano lleva en su ser y se exteriorizan en el actuar, en el comportamiento social; 
además los considera como una cualidad deseada en el ser humano; en tal sentido Arnold, (1991) plantea que los valores se pueden caracterizar por ser cualidades que le dan sentido al ser humano y valor a las relaciones sociales. Para Heidegger (1966) y Jeremías (1985) al hablar de objetividad de valores como aquellos que se pueden observar en el comportamiento del individuo hay que tener presente aspectos subjetivos, pues todos los valores que posee una persona son determinados por los sentimientos, ideología, cultura, historia personal, formación, creencias y estilo de vida de cada ser humano.

Existe una diversa taxonomía sobre los valores; Pilay (2013) los clasifica en valores naturales, los mismos que se conforman con las necesidades que posee una persona de sobrevivencia y de necesidades básicas, como la protección de los recursos; valores económicos, los que permiten garantizar la vida del ser humano en el medio en donde se encuentra; valores políticos son las relaciones y forma de vivencia que tiene una persona con la sociedad, tal como la paz, la justicia; valores morales y éticos, considerados como los que se dedican a satisfacer o caracterizar la dignidad e integridad de una persona como es la honestidad; y por último, los valores estéticos que se relacionan con el sentido de la belleza y armonía que posee la persona.

Camps (1994): manifiesta que los valores son adquiridos hasta convertirse en hábitos; es decir que los valores no son innatos, se van construyendo a partir de las experiencias y relaciones sociales.

La colaboración vista desde una perspectiva axiológica refleja un estado de actitud de una persona hacia sus relaciones con los demás y en otro sentido, puede ser visualizada como el medio para poder fomentar y desarrollar los valores.

En el momento de definir la colaboración, hay que considerar lo expresado por Campo (2016), al suponer que para que exista, se necesita el trabajo colectivo, tanto en lo científico, 
lo artístico, lo educativo o lo comercial; reconociendo las acciones necesarias para poder aprender y respetar a las otras personas.

El trabajo colaborativo en el ámbito educativo según Johnson (1999) es la unión de formas para enseñar o entrenar en un fin determinado a un grupo de personas.

Así mismo al referirse a la cooperación se hace mención al trabajo que es realizado por varias personas para cumplir un fin específico y particular que beneficia a todos de la misma manera, no existen las rivalidades ni las competencias laborales, escolares o personales, porque son un equipo y el bien es común; además esta ayuda se da por lo general de forma desinteresada (Abad, 2006).

Una estrategia didáctica para fomentar la integración y la colaboración entre estudiantes en el fortalecimiento de los valores humanos.

Todos los aspectos anteriormente analizados constituyen el precedente teórico tenido en cuenta para el diseño de una estrategia didáctica que permita fomentar la integración y la colaboración entre estudiantes. Además, se asume lo planteado por Ortiz y Mariño (2004), quienes expresan que en la concepción de una estrategia didáctica deben considerarse los siguientes aspectos:

- "La complementación de los roles del profesor y los alumnos a partir de considerar que ambos se enfrentan a un proceso en que enseñan y aprenden.

- La consecución de una actitud mental consciente de estudiante y docentes cuyos rasgos se integren a partir de las siguientes líneas de confluencia: un pensar positivo, un aprendizaje como experiencia agradable y satisfactoria.

- La compaginación del trabajo individual y en grupos. (Trabajo cooperativo)

- La búsqueda de un aprendizaje significativo a partir de la elaboración de estructuras estables y sólidas de conocimiento con actitudes tendentes a la flexibilidad. 
- $\quad$ La potenciación de la autorregulación y autocontrol de los estudiantes.

- La priorización del elemento cualitativo en la apropiación de los conocimientos, habilidades y valores" (Ortiz \& Mariño 2004, p.13).

Siguiendo estos lineamientos se proponen para el diseño de una estrategia didáctica las siguientes acciones:

1. Plantear objetivos de aprendizaje, concientizados por los estudiantes, en correspondencia con sus necesidades, intereses y motivaciones.

2. Organizar el trabajo de los estudiantes en grupos o equipos para facilitar el intercambio, la colaboración y cooperación, donde el papel del docente sea fundamentalmente de orientador.

3. Crear las condiciones para favorecer el aprendizaje de los estudiantes, definiendo las condiciones, interacciones entre los estudiantes y el profesor, contenidos del currículum y materiales didácticos.

4. Enfrentar a los estudiantes con tareas, solución de problemas, trabajos investigativos que propicien la colaboración y cooperación entre los sujetos del proceso de enseñanza-aprendizaje.

5. Incluir mecanismos de autoevaluación, control y evaluación a través de una diversidad de técnicas que incidan positivamente en el fomento de valores tales como: justicia, honestidad, veracidad, crítica y autocrítica.

Entre los principales problemas interculturales de la sociedad o manifestaciones de conductas antisociales en el ámbito escolar se encuentran: la discriminación, el mal comportamiento, la falta de respeto, violación de las normas de conducta fuera y dentro del aula. 
Mediante estrategias didácticas que promuevan el trabajo colaborativo se logrará la integración y cooperación entre los estudiantes lo que contribuye así al fomento de valores, estas estrategias deben contener las siguientes acciones:

1. Plantear objetivos de aprendizaje, concientizados por los estudiantes, en correspondencia con sus necesidades, intereses y motivaciones.

2. Organizar el trabajo de los estudiantes en grupos o equipos para facilitar el intercambio, la colaboración y cooperación, donde el papel del docente sea fundamentalmente de orientador.

3. Crear las condiciones para favorecer el aprendizaje de los estudiantes, definiendo las condiciones, interacciones entre los estudiantes y el profesor, contenidos del currículum y materiales didácticos.

4. Enfrentar a los estudiantes con tareas, solución de problemas, trabajos investigativos que propicien la colaboración y cooperación entre los sujetos del proceso de enseñanza-aprendizaje.

5. Incluir mecanismos de autoevaluación, control y evaluación a través de una diversidad de técnicas que incidan positivamente en el fomento de valores tales como: justicia, honestidad, veracidad, crítica y autocrítica.

\section{La gestión cultural de la identidad}

La Universidad tiene, pues, por razones históricas y normativas, una tercera función que sintéticamente podemos identificar como cultural y que debe ser considerada como una misión esencial.

La Universidad es la sede de la ciencia y de la razón. De aquí derivan cuatro características que deben determinar la cultura que la Universidad crea, promueve y difunde: 
- $\quad$ Científica: una cultura del logos frente al prejuicio; de la evidencia y la argumentación; en última instancia, sometida al método.

- Crítica: una cultura que se basa en la mejor tradición de la sospecha, de la puesta en cuestión de las visiones hegemónicas que legitiman relaciones de poder.

- $\quad$ Creativa e innovadora: en el sentido de que promueve la novedad relevante para mejorar la dignidad y la calidad de la vida humana.

- $\quad$ Académica: que opera en el nivel de síntesis, de interrelación y coordinación de saberes en una era en que los problemas son globales y transfronterizos.

La gestión de la formación cultural universitaria requiere de una perspectiva humanística caracterizada por:

- $\quad$ Poseer un conocimiento general de obras de la cultura nacional y universal

- Desarrollar su sensibilidad y gusto estético.

- Desarrollar una amplia competencia comunicativa.

- Desarrollar un pensamiento flexible y receptivo.

- $\quad$ Fomentar el hábito de lectura.

- Facilitar la creatividad y el dinamismo.

Otros rasgos que deben caracterizar a un educando universitario con un alto nivel de formación cultural:

- Tener un saber general, esencial y sintético sobre áreas fundamentales de la realidad.

- $\quad$ Poseer un espíritu abierto, indagador, exploratorio.

- Realizar una asimilación consciente, activa y crítica de la información.

- Apertura a los valores de todos los pueblos.

- $\quad$ Establecer nexos y relaciones entre los conocimientos. 
- Aplicar los conocimientos a la vida, vincular la teoría con la práctica.

- Asumir una participación creadora y transformadora de la sociedad.

- $\quad$ Poseer una formación ciudadana

El proceso formativo de los estudiantes supone la preparación en la universidad de un profesional que actúe acorde con los valores más genuinos de la nación, desarrolle habilidades que garanticen un desempeño competente, de excelencia académica con una alta productividad científico- tecnológica, además de un horizonte cultural válido para satisfacer las necesidades del país y a la vez insertarse en el escenario internacional.

La formación es, por tanto, un proceso que abarca a la personalidad en su integridad, es decir, comprende tanto lo cognitivo-instrumental como lo afectivo motivacional y volitivo, traducido en comportamientos. Ella constituye un crecimiento en profundidad que se realiza en el tiempo y no en segmento reducido de la vida, y esa realización es, además, el resultado de la conjugación de individual y lo colectivo; no se da en el aislamiento sino en la interacción social. (González Morales, 2003)

Una primera aproximación a tan importante proceso nos conduce a afirmar que el mismo se gestiona a partir de la labor extensionista de la Universidad, estructurada en una tríada con los procesos docentes e investigativos, que permita compartir cultura, conocimientos y comportamientos, lo cual posibilita potenciar y promover los valores más trascendentales de la sociedad, fortaleciendo su identidad y toda la riqueza histórica, económica y social de la misma. Al mismo tiempo, incluye las necesidades e intereses culturales a partir del conocimiento que tienen los propios educadores y promotores en la comunidad universitaria.

El proceso de interacción de la Universidad con la comunidad, en la misma medida que abre nuevos canales para la promoción cultural desde el propio quehacer universitario, 
hace partícipe a la población del disfrute en términos de consumo de los principales avances alcanzados por la institución en el proceso de formación, investigación y extensión.

Podemos acercarnos a una definición de extensión universitaria entendida como el proceso de comunicación entre la universidad y la sociedad, basado en el conocimiento científico, tecnológico, cultural, artístico, humanístico, acumulado en la institución y en su capacidad de formación educativa, con plena conciencia de su función social (Fernández,1981).

“...la extensión universitaria es el proceso que tiene como propósito promover cultura en la comunidad intra y extrauniversitaria como parte de la contribución universitaria al desarrollo cultural. Se parte del criterio de que las distintas concepciones de la extensión, implican relaciones diferentes de la universidad con la sociedad, sus organizaciones e instituciones, así como diferentes valoraciones en torno a la universidad, al saber y a la relación que se instaura entre esta y los diferentes sectores sociales involucrados" (González, 2013

Esta perspectiva amplía la integración entre universidad y sociedad, entre oferta y demanda de conocimientos, entre lo que se investiga y los problemas de la sociedad, para dar lugar a un proceso interactivo donde el conocimiento se construye en contacto permanente con su medio y es permeado por el mismo.

\section{La interculturalidad en la universidad}

Walsh (2005) asocia la interculturalidad en América Latina muy apegada a la política educativa para los pueblos indígenas, por las Organizaciones No Gubernamentales (ONG) o por el mismo Estado. Ello destaca, en el caso ecuatoriano, una centralidad en las diferencias de idiomas hecho que exige la realización de un cambio en el rediseño del currículo, pues la afluencia de hablantes nativos se incrementa cada vez más en las aulas de clases, planteando a 
los profesores solucionar este conflicto mediante la transformación de sus metodologías, actitudes, valores que ayuden a asumir la complejidad escolar (Romera, 2015). Resolver este asunto significa además insertarse en las coordenadas del mundo global.

Este contexto introduce la variable que representan las migraciones forzadas, refugiados políticos y fronteras políticas militarizadas y también estudiantes (Grimson, 2001), esas peculiaridades generan necesidades económicas, políticas, costumbres de países o ciudades diferentes que crean conflictos a solucionar, entre los cuales se encuentran los reclamos sobre el reconocimiento cultural de los grupos indígenas o emigrantes quienes demandan equidad con sus hábitos culturales.

La educación de diversas culturas es algo que ha existido desde hace mucho tiempo atrás, según Turra (2009), comienza a desarrollarse a mediados del siglo XX lo cual reconoce la interculturalidad en la educación; como tema mantiene su vigencia, aunque algunas sociedades han intentado erradicar en cierta forma la interculturalidad por el hecho de que no se adaptarían a sus normas y costumbres. Ante esta postura otras sociedades reclaman la importancia que representan pueblos con diversas costumbres incorporados a la convivencia cultural lo que aporta riqueza cultural universal. Este cambio determina que la ciencia social en los años ochenta se desarrollen estudios sobre interculturalidad con enfoque interdisciplinario (Baz, 2003).

En el contexto de Ecuador algunos instrumentos como la declaración de los Derechos Humanos (1948) hasta la Ley Orgánica de Educación, (2006), ha existido una continua referencia explícita a la igualdad de oportunidades independientemente de la nacionalidad de origen y esto ha supuesto una protección jurídica de gran interés para llevar adelante actuaciones concretas (Rodríguez \& otros, 2011). Esa dimensión legal se corresponde con realidades en las cuales se alude a un "despertar étnico" de 1992, con el cual los pueblos originarios y las comunidades afrodescendientes del continente americano reclaman no 
solamente el derecho a la tierra y al territorio, a sus lenguas y sus culturas, sino también el derecho a una educación cultural y lingüísticamente oportuna (Cortés, 2002). En el ámbito pedagógico, esta demanda por el reconocimiento de la diferencia y su inclusión activa en las políticas educativas adopta denominaciones multiculturales. En el contexto latinoamericano desde los años setenta se observa la necesidad de ofrecer una educación diversificada y apropiada para los contextos indígenas y afrodescendientes.

Este trabajo concreta la finalidad de gestionar la interculturalidad en los salones de clases como interacción de modos de vida y pensamientos que significa además una reestructuración social desde una perspectiva cultural, hecho que obliga a determinar propuestas estratégicas que permitan actuaciones enfrentadas a esta situación y otras que puedan producirse. Se ancla en la visión de que la interculturalidad es "un problema pedagógico" (García, 2009), con mucha presencia en varios países del mundo en el ámbito educativo; posición que responde a la percepción de un incremento notable de estudiantes extranjeros emigrantes que proceden de etnias indígenas y contribuyen al pluralismo cultural en el contexto en el que se encuentran, esta autora lo mira también de un lado positivo al señalar que favorece la creación de un "nuevo signo de identidad de la sociedad democrática moderna, constituye una verdadera oportunidad de avance hacia mentalidades más tolerantes y abiertas" (2009). Coincidente con esta postura Martín Barbero (2009) acota que la mundialización cultural contribuye a las condiciones de igualdad cultural e intercambio dentro de su país.

En cambio Ortiz (2007) muestra que la interculturalidad es reconocer la diferencia que existe en un grupo de personas, y convivir con ellas, estas diferencias pueden ser entre los pueblos indígenas con el propósito de resaltar los problemas sociales en el lugar determinado en donde conviven, estos problemas sociales se deben resolver en base a los valores, en ética 
y una buena moral para así poder ser equitativos con todos y poder construir una sociedad con coherencia y armonía.

Los puntos de vista anteriormente descritos son las ideas que sostienen la propuesta que desarrollamos visto cual modelo de convivencia intercultural entendida desde el modo de satisfacer las necesidades de los estudiantes cuyos rasgos diferentes, los hacían objeto de exclusión y colocación en un lugar subalterno, razones que obligan colocar un modo evolucionado de atención a la diferencia inclusivo respetuoso y justo.

Para Davini (2008), los modelos son el producto de "experimentos de laboratorio", aislados, cuya distancia con la realidad motiva sean desestimados, ya que se piensan cual teoría y no en su eficacia metodológica, causa que conduce a los docentes a despreocuparse del tema. Interesa acotar que ellos representan una visión teórica que eleva al rango de categorías esenciales aquellos conceptos que están en el centro del núcleo de la teoría explicando sus relaciones (Leyva, 2009). Por tales motivos la interculturalidad será el principio categorial con el cual se valorará el sistema de relaciones que debe ser establecido en el aula pluricultural.

El punto de vista que se asume sobre este principio es que la interculturalidad es un acto de interacción entre los grupos culturales la que configura relaciones basadas en el respeto y la igualdad (Schmelkes, 2006), las mismas constituyen una nación beneficiándola en muchos aspectos. Lo intercultural se manifiesta no sólo en el ámbito de conocimiento, sino en su disposición, en el modo de hacer las cosas, lo cual se visiona cual horizonte cultural de donde el sujeto extrae hábitos para comprender o reconocimiento los instrumentos que le producen gratificación mediante otros saberes. Por esta razón, el diálogo cultural ésta más allá de una postura ética o normativa, es un modo de consentimiento frente al mundo.

De esta postura normativa Zimmermann, (1997), extrae los modos de inclusión en los centros educativos a promover como forma de relaciones: 
- Es una consecuencia lógica de los derechos humanos y una lógica de la ética que no admite a otros el derecho de autodeterminación cultural, nunca podría argumentar en contra de una política discriminatoria de su propia cultura/lengua.

- Hay que mencionar también un argumento económico. La economía requiere recursos intelectuales (humanos).La mayoría gasta sus esfuerzos en una actividad semi-asimilacionista y semi-resistente.

- Las relaciones interétnicas e interculturales de mutuo respeto y dignidad que proporcionan una identidad positiva a cada uno y una autoestimación, disminuyen la posibilidad de futuros conflictos, que son dañinos para la vida social en general y para la economía en particular.

Para un salón donde tenga lugar la educación intercultural es requisito la extensión de culturas y el status de las personas por igual, aspiración a lograr mediante la autoestima de los estudiantes, que es la fuente de su confianza, firmeza al tomar decisiones e identificar su identidad, para que se construya un ambiente de paz, y justicia en la convivencia (Johansson, 1997). Por esto se propone utilizar el método inductivo intercultural que, según explica Sartorello, (2016) no es un método tradicional de reglas rígidas en el aula, resulta lo contario, ya que se basa en lo epistémico y pedagógico integral para generar aprendizajes socioculturalmente significativos a partir de las actividades sociales"; un método utilizado por Jorge Gasché para el "Programa de Formación de Maestros Bilingües de la Amazonía Peruana (FORMABIAP)"

Un método que busca generar un modelo de participación donde profesores y estudiantes coincidan en actividades sociales, productivas, rituales y recreativas, practicadas en las comunidades indígenas, acciones que se tornan fuentes principales para la elaboración y fortalecimiento del conocimiento indígena, el quehacer cotidiano, que se practica muchas 
veces de manera automática (Silva, 2012). Los momentos metodológicos del modelo se sintetizan en: listar las principales actividades tradicionales de la comunidad; organización y descripción de las actividades seleccionadas (por ejemplo, se separan las actividades de hombres y mujeres, de adultos y niños).

La temática elegida se traduce en contenidos concretos de la realidad de cada comunidad, lo que puede articularse con los conocimientos científicos del currículo aptos para ser trabajados en las escuelas indígenas u otras culturales, temática escogida en base a un trazado filosófico y político, ejecutada como un conjunto de conceptos necesarios para producir contenidos. Este metodología se utilizará para realizar actividades sociales que se desarrollen a partir de los miembros adultos de la comunidad y se realizará a lo largo de las diferentes temporadas del año, las cuales guardan correspondencia con los valores de las culturas nativas y que en tanto método aspira a revalorizar la sociedad y cultura indígena a través de la comprensión antropológica en un lenguaje accesible" (Gasché, 2013). El idioma nativo de donde conviven, se utilizará con la intención de que al expresarse haya una mayor aceptación por el grupo y un mejor aprendizaje para ellos, en un proceso que fomenta la participación activa en la escuela

Hay que recalcar que estos apartados del lenguaje nativo al sistematizarse se erige como un redimensionamiento de los conocimientos linguiísticos del otro significativo, quien egresa de la escuela con una cultura idiomática superior al desarrollarse un aprendizaje del español mediante el contraste o la comparación.

La adopción de un modelo comparativo es un procedimiento inclusivo y requiere la colaboración de las familias por medio de la información oportuna de las ventajas que aporta la enseñanza de la lengua por contraste y su currículo superior. Otra idea que debe estar en la base de las prácticas educativas es que todos los niños y niñas pertenecen a un grupo, y por tanto, todos pueden aprender en la vida normal del mismo. Esto implica concebir el aula 
como una comunidad de aprendizaje y convivencia, donde el alumnado comprende esta peculiaridad de la orientación educativa. Hablar de educación inclusiva va a suponer un aprendizaje meso-social, es decir en su grupo. Los tiempos de estancia en estas aulas/programas serán los “mínimos e imprescindibles". Se trata, en definitiva, de llevar el aprendizaje de la lengua vehicular al aula ordinaria (González; 2012).

Las demandas educativas de la diversidad cultural exige el desarrollo igualitario tanto en el primer año de educación inicial hasta la universidad, una demanda de estrategias formativas, intervención e inclusión y que tome en cuenta los problemas que se vivencian día a día en cada comunidad, de forma que se logre el conocimiento contextualizado. Al respecto la estrategia que se propone emerge de la necesidad que tienen estas personas de comunicación correcta en el contexto donde se encuentran, capacitarlos resulta la creación de vínculos comunicativos para la colectividad, participación que no diferencia ni excluye ningún nivel de la estructura social para poder contribuir con la propuesta (Giménez, 1997).

\section{Conclusiones}

$\mathrm{Al}$ aportar un nivel reflexivo que brinde guías para conocer el sentido y el valor de la actividad intelectual desde la identidad en la universidad, se enriquece el conocimiento de la concepción y a la vez de los escenarios de la academia. Este estudio ha demostrado entonces que el diálogo de lo epistemológico en las relaciones de la coherencia conceptual tiene su efecto en el contexto que lo especifica. Una aseveración que trascurre por las intersecciones que estructuran la ciencia cultural, para enriquecer al sujeto y disponerlo en condiciones de expresar sus sentimientos y racionalidades desde el compromiso con su realidad más inmediata.

En esa realidad emerge la funcionalidad histórica que da sentido y finalidad a la vida de una sociedad, pues viene desde las diferentes generaciones, estableciendo un vínculo entre 
pasado, el presente, y el futuro. En el entorno de la identidad se impacta la diversidad cultural, un factor que por la labor de instituciones y organismos ha sido posible recuperar aquello que se daba por perdido para poder apreciarlo y valorarlo. La Universidad es la sede de la ciencia y de la razón con características determinantes de la creación, la promoción y difusión de relaciones interétnicas e interculturales de mutuo respeto y dignidad que proporcionan una identidad positiva a los sujetos sociales y una autoestimación, que neutraliza la posibilidad de conflictos dañinos para la vida social.

\section{Referencias Bibliográficas}

Abad M. \& Benito, M .L. (2006): Cómo Enseñar Juntos a alumnos diferentes: Aprendizaje Cooperativo. Zaragoza: Egido.

Ambrosi, A., Peugeot, V. \& Pimienta, D.(2005), Palabras en juego: Enfoques multiculturales sobre las sociedades de la Información. Francia:C \& F Éditions.

Arbat (2012), La Importancia de la colaboración, Bilbao: Arbatbilbao.

Arias, N. M., Arraigada, P.C., Gavia, H. L, Lillo, M. L. \& Yánez, G. N. (2009). Integración Escolar Universidad de Chile. Santiago. Chile. Disponible en: Http://Www.Tesis.Uchile.Cl/Tesis/Uchile/2005/Arias_I/Sources/Arias_I.Pdf

Arias, N. M., Arraigada, P.C., Gavia, H. L, Lillo, M. L. \& Yánez, G. N. (2009). Integración Escolar Universidad de Chile. Santiago. Chile. Disponible en: Http://Www.Tesis.Uchile.Cl/Tesis/Uchile/2005/Arias_I/Sources/Arias_I.Pdf 
Arnold, P. J. (1991). Educación, Valores, Propósitos y Objetivos En Educación Física, Movimiento y Curriculum. Madrid:Morata.

Bayardo, R. (2001). Antropología, Identidad y Políticas Culturales. Programa Antropología de la Cultura ICA, FFyL, Universidad de Buenos Aires. Recuperado de: bayardo@tropos.filo.uba.ar.

Campo, L. (2006). Colaboración Versus Cooperación. Santa María, España. Disponible en: Https://Lcampolorenzana.Wordpress.Com/2006/12/30/Colaboracion-VersusCooperacion/

Camps, V. (1994). Los Valores De La Educación. Madrid: Alauda Anaya.

Davini, M. C. (2008). Métodos de enseñanza. Didáctica general para maestros y profesores. DOI: http://ciequilmes.com/wp-content/uploads/2013/03/1-davini-metodo.pdf

Fernández Varela, Jorge; Puga, Domingo y Tünnerman, Carlos. (1981) Notas sobre la conceptualización de la Extensión Universitaria. México. Universidad Nacional Autónoma de México. Cuadernos de Extensión Universitaria. México.

Gasché, J. (2013). Éxitos y fracasos de una propuesta educativa basada sobre el" Método Inductivo Intercultural" implementado en el Perú, México y el Brasil. ISEES: Inclusión Social y Equidad en la Educación Superior, (13), 17-34.

Giménez, Gilberto. (1994). Comunidades primordiales y modernización en México. En: Giménez, G. \& Pozas, R. (eds): Modernización e identidades sociales, pp. 151-183. México: UNAM.

Giménez, C. (1997). La naturaleza de la mediación intercultural. Migraciones. Publicación del Instituto Universitario de Estudios sobre Migraciones, (2), 125-159. 
(2002). Identidades sociales, identidades étnicas. En: Eugenio Alcaman et al.

Interculturalidad, Sociedad Multicultural y Educación Intercultural, pp. 31-55.

México: Castellanos Editores, Asociación Alemana para la Educación de Adultos, Consejo de Educación de Adultos de América Latina.

Giménez, G. (2012). La cultura como Identidad y la identidad como Cultura. Recuperado de://https://estudioscultura.wordpress.com/2012/03/13/

González de Alba, V. (2013). Valores humanos. Universidad Central de Chile. Revista Latinoamericana de Educación Inclusiva. 6(2) Disponible en: Http://Www.Rinace.Net/Rlei/Numeros/Vol6-Num2/Rlei\%206,2.Pdf

González, M. J. A. (2012). Las aulas de inmersión lingüística para alumnado inmigrante en el marco de la escuela inclusiva: algunas propuestas de mejora. Tendencias pedagógicas, (19), 25-42.

González Fernández-Larrea, Mercedes; González González, Gil. (2013) ¿Extensión universitaria, proyección social o tercera misión? una reflexión necesaria. Revista Congreso Universidad. Vol. II, No. 2, Editorial Universitaria Félix Varela, La Habana.

González Morales, Alfredo. (2003) La formación humanística cultural en la educación cubana. Ponencia presentada en la I Conferencia Internacional de Estudios Humanísticos. Universidad Central "Martha Abreu "de Las Villas.

Heidegger, M. (1966). Carta sobre Humanismo. Taurus. Madrid, España

Jeremías, J. (1985). Teología del Nuevo Testamento. Salamanca: Sígueme. 
Jujuy (2015). La importancia de la cultura para la sociedad. San Salvador de Jujuy, Argentina. Disponible en: http://www.jujuyalmomento.com/post/40898/la-importancia-de-lacultura-para-la-sociedad

León, G.M.(2012), Importancia de la Diversidad Cultural y Étnica .San Antonio de Cajamarca. Perú. Disponible en: http://es.slideshare.net/mariagracialeon/importanciade-la-diversidad-cultural-y-tnica

MOSEIB. (s.f.). Recuperado el 2017, de https://educacion.gob.ec/category/educacionintercultural-bilingue-i/

Montaluisa, D., \& Sanchez, C. A. (Junio de 2016). El Rol de Docente en el Rescate de la identidad cultural. Identidad Cultural. Latacunga, Cotopaxi, Ecuador.

Odello, M. (1012). El Derecho a la Identidad Cultural de los pueblos Indigenas de América. México.

Ortiz, E. \& Mariño, M. (2004). Estrategias educativas y didácticas en la educación superior, Pedagógica Universitaria. Revista electrónica de la dirección de formación de profesionales, Ministerio de Educación Superior, 9(5), 25 -26.

Padrino, M. A. (2009). Valores. Ministerio del Poder Popular para la Educación Unidad Educativa Instituto Jesús Señor Zaraza - Estado Guárico República Bolivariana de Venezuela.

Pere, P. M. (2009). Aprendizaje Cooperativo y Educación Inclusiva: Una Forma Práctica de Aprender Juntos Alumnos Diferentes. Universidad de Vic. Barcelona. España. Disponible en: Http://Www.Mecd.Gob.Es/Dms-Static/F4d240d3-55ad-474f-Abd7Dca54643c925/2009-Ponencia-Jornadas-Antiguas-Pere-Pdf. 
Pérez, P. J. \& Merino, M. (2011). Definición de Colaboración. Disponible en: Http://Definicion.De/Colaboracion/

Pilay, T. F. (2013). Enseñanza de los valores humanos. Clasificación de los valores humanos. Quito. Ecuador.

Romero, P. K. (2013). Valores y principios humanos. Cartagena, Bolívar Disponible en: http://es.slideshare.net/kevinromeroperez56/valores-y-principios-humanos.

Saez, G. (2013). Estrategia Didáctica Dirigido a la formación de la Identidad Cultural en los estudiantes de Secundaria Básica. La Habana, Cuba: Editorial Universitaria.

Sánchez, M., \& Dalama, M. (2012). "Identidad Cultural Latinoamericano desde la Perpectiva de Josè Marti". Eumed.net. America: Juan Carlos M. Coll.

Sartorello, S. C. (2016). Política, epistemología y pedagogía: el Método Inductivo Intercultural en una escuela tseltal de Chiapas, México. LiminaR, 14(1), 121-143. DOI: http://www.scielo.org.mx/scielo.php?

Silva, L. J. (2012). El método inductivo intercultural y el calendario socioecológico como estrategias para el fortalecimiento de una formación crítica e intercultural de profesores indígenas de los estados de Minas Gerais y Bahía, Brasil. ISEES: Inclusión Social y Equidad en la Educación Superior, (10), 79-96.

Stainback, S. B. (2001). «Components Crítics en el Desenvolupament de L’educació Inclusiva». A Suports. Revista Catalana D’educació Especial I Atenció a .la Diversitat. 5 (1), 26-31. UNESCO. (s.f.). Obtenido de https://www.google.com.ec/?hl=es\&gws_rd=cr\&ei=SabUWKWqJeGXjwT4z5ygDQ\# $\mathrm{hl}=$ es\&q=identidad+cultural+unesco++ecuador $+\& *$ 
UNESCO (2004). División de Políticas Culturales y Diálogo Intercultural, Declaración Universal de la UNESCO sobre la Diversidad Cultural.

UNESCO (2013). Declaración Universal de la UNESCO sobre la Diversidad Cultural Nacionalidades y Grupos Étnicos Ecuador. ECUADOR: País Plurinacional e Intercultural. Quito. Ecuador en: http://gruposetnicosecu.blogspot.com/2013/04/ecuador-pais-plurinacional-e.html.

Valencia, A. (2012), Educación Especial. Universidad de Antioquia. Antioquia. Colombia. Disponible en: Http://Eduincluye.Blogspot.Com/2012/10/Historia-De-La-InclusionEducativa_6152.Html

Vergara Estevez, J. (s/f). Cuatro tesis sobre la identidad cultural latinoamericana. Una reflexión sociológica. Revista de Ciencias Sociales (Cl), núm. 12, 2002, pp. 77-92. Universidad Arturo Prat. Tarapacá, Chile. Disponible en: http://www.redalyc.org/articulo.oa?id= 70801206.

Zimmermann, K. (1997). Modos de interculturalidad en la educación bilingüe. Reflexiones acerca del caso de Guatemala. Revista Iberoamericana de Educación, 13, 113-127. 